\title{
Detection of HOXA1 Expression in Human Breast Cancer
}

\author{
Alain Chariot*, ${ }^{*}, 1$ and Vincent Castronovo $\dagger$ \\ *Department of Clinical Chemistry and Clinical Oncology, †Metastasis Research Laboratory, University of \\ Liege, Belgium
}

Received March 28, 1996

\begin{abstract}
Homeodomain-containing proteins are transcription factors that regulate the coordinated expression of multiple genes involved in development, differentiation and malignant transformation. To better understand the role played by these proteins in breast cancer cells, we demonstrate, using semi-quantitative RT-PCR experiments, that progestin induces HOXA1 mRNAs in MCF7 cells. This is the first evidence of regulation of a HOX gene by steroids. Moreover, we detected HOXA1 expression in a variety of human breast cancer lesions, suggesting that HOXA1 may be required for the establishment of breast cancer cells phenotype. We propose that HOXA1 gene could be one of the orchestrators that regulate breast epithelial cell differentiation and that alteration of HOXA1 expression could play a role in breast cancer progression. () 1996 Academic Press, Inc.
\end{abstract}

Cell transformation and tumor progression are associated with coordinated activation an inhibition of specific intact or altered genes $(1,2)$. This complex genetic program involves proteins that control cellular proliferation and/or differentiation. Transcription factors implicated in organogenesis are ideal candidates to regulate these multistep processes.

Homeodomain-containing proteins act, as transcription factors, in the genetic control of genes involved in development and differentiation (3). These genes were initially discovered in Drosophila where they control segment identity (4). Homologous homeobox genes have been since cloned in multiple species, including human $(5,6)$. To date, 38 human class I homeobox genes have been discovered. They are organized in four clusters (HOX loci A, B, C and D), located respectively on chromosomes 7, 17, 12 and 2 (7).

There is accumulating evidence suggesting a possible involvement of HOX genes in malignant transformation. A potential role of these genes in neoplasia was first documented in leukemia, as reviewed (8). Distinct chromosomal translocations or incorporation of a regulatory element in the vicinity of a homeobox gene confers an oncogenic potential to the protein product in hematopoietic cells $(9,11)$. Moreover, alterations of HOX expression have been also detected in a variety of human tumors (12-15). In addition, ectopic HOX expression obtained by transfections of Hox genes into murine and rat cells induces tumorigenicity (16). This oncogenic potential has also been attributed to divergent homeobox genes that are not included in the 4 clusters (17-20).

As a first step to investigate the role played by HOX genes in breast cancer cells, we have previously cloned three alternatively spliced HOXA1 transcripts from a MCF7 cDNA library and demonstrated that HOXA1 expression was modulated by retinoic acid in these cells (21). In this study, we further demonstrate that these HOXA1 transcripts are induced by progestins, another differentiating agent, in MCF7 cells. Moreover, we systematically detected HOXA1 expression in a variety of human breast cancer lesions as well as in the adjacent tissues. Taken together, our results suggest that HOXA1 may play a key role in breast epithelial cell differentiation and may be associated with breast cancer phenotype.

\footnotetext{
${ }^{1}$ Correspondence should be addressed to Alain Chariot, Clinical Chemistry, Pathology, B23, 3rd floor, Sart-Tilman, Liège, 4000 Belgium. Fax: 3241668823.

Abbreviations used: RT-PCR, reverse transcriptase polymerase-chain reaction; Class I, homeoproteins sharing an Antennapedia-like homeodomain; DMEM, Dulbecco's modified Eagle medium.
} 


\section{MATERIALS AND METHODS}

Cell culture and treatment. MCF7 cells were derived from the pleural effusion of a patient with breast adenocarcinoma and were obtained from the American Type Tissue Collection (Rockville, MD). The cells were maintained in "DMEM" containing Dulbecco's modified Eagle medium with 10\% fetal calf serum (Gibco, Grand Island, NY) and antibiotics (100 $\mu \mathrm{g} / \mathrm{ml}$ ). Before any treatment, MCF7 cells were grown in phenol red free DMEM/HAM F-12 (Sigma Chemical Co., St Louis, MO) for 24 hours in order to eliminate interfering steroids. ORG 2058 (Amersham, Airlington Heights, IL) was added to the cultures as concentrated stock solutions in pure ethanol $\left(0.1 \%\right.$ of the total volume) to raise a final $10^{-8} \mathrm{M}$ concentration. Untreated cells were exposed to the same amount of ethanol.

Tissue specimens. Breast cancer samples as well as normal adjacent tissues were collected from patients treated for surgery (Hospital Clinic, Villarroel, 170, Barcelona, Spain) and were immediately frozen in liquid nitrogen.

HOXA1 and actin cDNA synthesis. Total cellular RNA was isolated by the guanidium isothiocyanate extraction procedure and cesium chloride gradient centrifugation (22). cDNA synthesis was performed at $42^{\circ} \mathrm{C}$ for 30 minutes using $1 \mu \mathrm{g}$ or $0.1 \mu \mathrm{g}$ total cellular RNA (for HOXA1 and actin cDNA synthesis respectively) using Superscript reverse transcriptase (Gibco BRL, Gaithesburg, MD) and a 3' specific primer (primer 3A, see Figure 1 for HOXA1 cDNA synthesis or 5'-CTGCATCGTGTCGAAGAG-3' for actin cDNA synthesis). The RNA template was degraded by RNase H (Gibco BRL). The cDNAs were then purified through GlassMAX Spin Cartridge (Gibco BRL). All reactions were performed in parallel in the absence of reverse transcriptase to verify dependence on an RNA template for subsequent PCRs.

Semi-quantitative PCRs. HOXA1 and actin cDNAs (see above) were amplified using either HOXA1 primers 5A and 3B (see Figure 1) or actin primers (5'-ATGATATCGCCGCGCTCG-3' and 5'-CGCTCGGTGAGGATCTTCA-3' (23)) 1ocated on different exons in both cases. HOXA1 and actin PCR amplifications included 35 and 25 cycles, respectively. The PCR products were submitted to Southern blotting using as $\left[{ }^{32} \mathrm{P}\right]$ labeled probe, HOXA1 (see Figure 1) or actin cDNA fragments. For semi-quantitative analysis, titration curves for both HOXA1 and actin cDNAs were prepared to determine the linear range of both amplifications (24). To avoid the plateau phase (see Figure 2), the semi-quantitative RT-PCR experiments were performed using $5 \mu \mathrm{l}$ of HOXA1 or actin cDNA solutions as templates. The intensities of hybridization signals were quantified by the Scan Analysis program for the Macintosh (Biosoft, Cambridge, UK).

Southern blotting. The PCR products were electrophoresed on a $1 \%$ agarose gel and blotted to a Qiabrane membrane (Qiagen, Chatsworth, CA) under alkaline conditions. Blots were hybridized with $\left[{ }^{32} \mathrm{P}\right]$ labeled HOXA1 and actin probes (see above), in $50 \%$ deionized formamide, $5 \times \mathrm{SSC}, 50 \mathrm{mM}$ Tris $\mathrm{pH} 7.5,10 \times$ Denhardt's $250 \mu \mathrm{g}$ salmon sperm DNA and $0.5 \%$ $\mathrm{SDS}$ at $42^{\circ} \mathrm{C}$ overnight and washed down to $0.1 \times \mathrm{SSC}, 0.5 \% \mathrm{SDS}$ at $65^{\circ} \mathrm{C}$ for $30 \mathrm{~min}$, twice. Films were exposed for 1 to 2 hours at room temperature.

\section{RESULTS AND DISCUSSION}

We have previously reported the molecular characterization of three alternatively spliced HOXA1 mRNAs from a MCF7 cDNA library (21). A map of the HOXA1 primary transcript structure is illustrated in Figure 1. There are two potential introns in HOXA1 gene: introns 0 and 1. These sequences (represented by white rectangles in Figure 1) can be alternatively spliced out to generate three distinct HOXA1 transcripts. Indeed, the shortest $2.0 \mathrm{~kb}$ HOXA1 mRNA encoding a truncated polypeptide is generated by splicing out both introns 0 and 1 whereas a second $2.2 \mathrm{~kb}$ transcript encoding the homeodomain-containing protein is obtained by splicing out intron 1 exclusively. Alternatively, a third $2.6 \mathrm{~kb}$ HOXA1 transcript has been identified: in this case, the primary transcript is not submitted to any splicing event. The resulting HOXA1 mRNAs presumably encode another truncated product.

\section{HOXA1 Is Modulated by Progestin ORG 2058 in MCF7 Cells}

The ability of steroids to affect HOXA1 expression in MCF7 cells was examined by performing semi-quantitative RT-PCRs using template RNAs extracted from cells treated with progestin ORG2058. To achieve this goal, RT-PCR experiments were performed using primers selected to amplify $5^{\prime}$ end HOXA1 cDNAs (see Material and Methods) as well as primers located on different exons of the actin gene to amplify $\beta$-actin cDNA as an internal control $(24,25)$. The resulting amplified fragments were analysed by Southern blots using either a HOXA1 or an actin specific probe (see Material and Methods). Three fragments of $1121 \mathrm{bp}, 655 \mathrm{bp}$ and $452 \mathrm{bp}$ corresponding to the three transcripts described above, were observed for HOXA1 Southern blots (Figure 2B). To get quantitative information from the RT-PCR experiments, a cDNA dose/response curve was established. As illustrated in Figure 2A and 2B, a linear production of amplified fragments was 
A

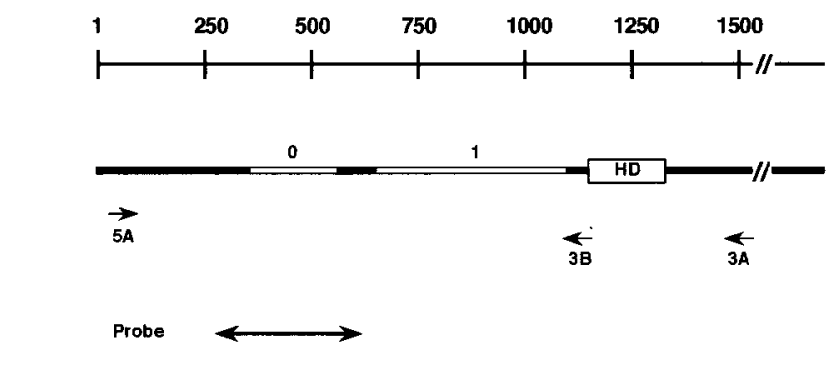

B

Primer 5A: 5'ATGAACTCCTTCCTGGAATA-3'

Primer 3A: 5'-GTTGTCTGGGGCTGGAGCC-3'

Primer 3B: 5'-CGTACTCTCCAACTTTCCC-3'

FIG. 1. A. On the heavy line below the numerical scale is a map of a HOXA1 primary transcript. Shown in white areas within this heavy line are potential introns 0 and 1 that are alternatively spliced out to generate distinct HOXA1 mRNAs. The homeodomain is represented by a white rectangle labeled "HD." The horizontal arrows represent primers to perform HOXA1 cDNA synthesis (primer 3A) and amplification of HOXA1 5' end cDNAs (primers 5A and 3B). The probe used to perform Southern blots is illustrated below. B. Sequences of the HOXA1 primers used in this study.

observed when 1 to $10 \mu \mathrm{l}$ of actin cDNA or 0.2 to $5 \mu \mathrm{l}$ of HOXA1 cDNA were added to the reaction mixture for PCRs. To avoid the plateau phase, the subsequent semi-quantitative RT-PCR experiments were therefore performed with $5 \mu \mathrm{l}$ of cDNA solutions.

HOXA1 and actin semi-quantitative RT-PCRs performed on MCF7 cells treated with ORG 2058 are illustrated on Figure 3A and 3B, respectively. All three HOXA1 transcripts were induced by $10^{-8} \mathrm{M}$ progestin ORG 2058 in these cells. The induction of HOXA1 expression was detectable within the first 24 hours of treatment and was still clearly increasing after 96 hours (see Figure 3A). Interestingly, we have observed that the transcript encoding a homeodomain-containing protein is preferentially induced during the first 48 hours of treatment whereas the shortest transcript encoding a truncated protein lacking the conserved DNA-binding domain is not as dramatically affected by such treatment during the first 48 hours (data not shown). As expected, the actin levels of expression remained constant in these cells (see Figure 3B). This original observation may allow a better understanding of the crucial role played by this HOX gene in breast cancer cells. Indeed, whereas it was clearly demonstrated that HOXA1 gene is a primary target for retinoic acid in distinct cell types such as embryonal carcinoma cells (26) and breast cancer cells, no information was available about possible HOX modulation by steroids. A recent study reported that expression of two murine Hox genes could respond to hormonal secretions (27). However, the steroid responsible for this modulation was not clearly identified. So far, the precise mechanism of HOXA1 induction by progestins remains totally unknown. To our knowledge, a potential hormoneresponsive element that can interact with the progesterone receptor (PR) still has to be identify in the HOXA1 promoter. Moreover, it is still unclear whether other HOX genes are modulated by steroids such as progestins and/or estrogens.

\section{HOXA1 Is Expressed in a Variety of Human Breast Cancer Lesions}

We further investigate HOXA1 expression by performing similar RT-PCR experiments on total RNAs extracted from a variety of human breast cancer lesions. As illustrated in Figure 4, we systematically detected HOXA1 expression in the investigated breast carcinoma samples. Com- 


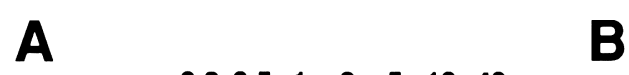

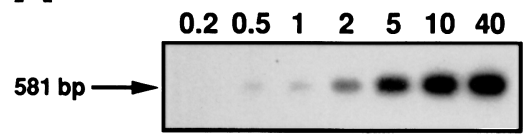

B ACTIN
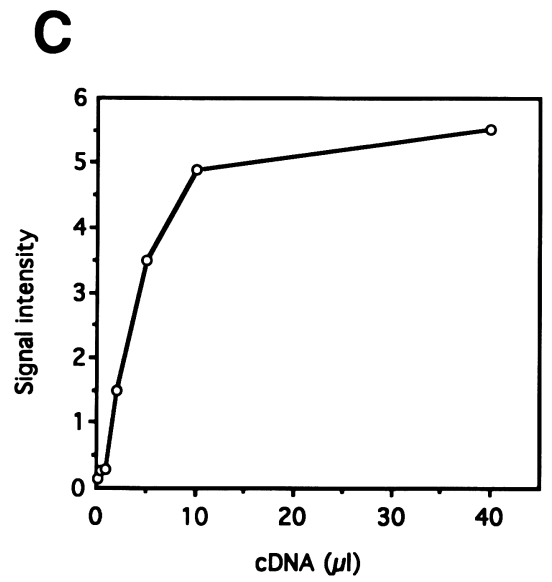

B

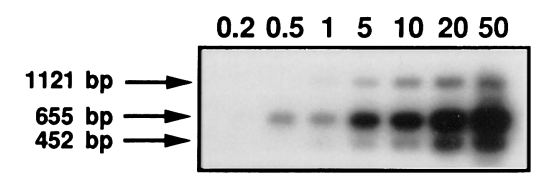

D

HOXA1

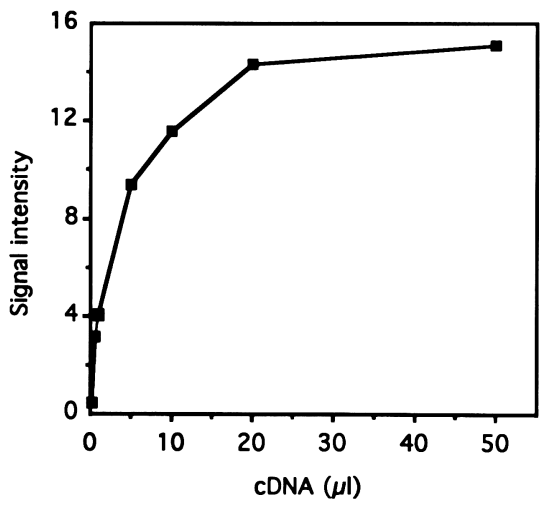

FIG. 2. Titration curves for both actin and HOXA1 PCR amplifications ( 25 and 35 cycles, respectively). Serial dilutions of actin or HOXA1 cDNAs were used as templates to perform PCR amplification, using appropriate HOXA1 specific primers. The resulting amplified fragments were subjected to Southern blotting. A. Autoradiogram of Southern blot performed with a $\beta$ actin cDNA probe. cDNA dilutions are indicated above the autoradiogram. B. Autoradiogram of Southern blot performed with a HOXA1 cDNA probe. C and D. Signal intensities of the amplified products were quantified by densitometry (arbitrary units) and plotted. Subsequent semi-quantitative RT-PCR experiments were performed using 5 $\mu \mathrm{l}$ of cDNA templates in both cases.

pared to the other transcript, the HOXA1 mRNAs encoding the homeodomain-containing protein harbor a higher level of expression in the investigated samples.

In the murine system, ectopic expression of Hox-a1 in transfected rodent cells induced tumorigenesis (16). Moreover, upregulation of the murine Hox-al gene has been recently seen in neoplastic mammary glands (27). Taken together, these studies suggest that HOX-a1 gene may play
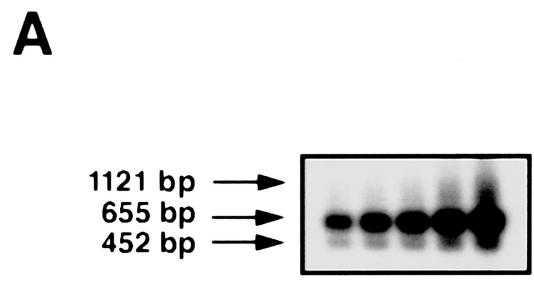

B

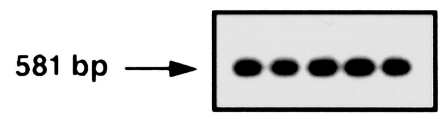

FIG. 3. HOXA1 and actin semi-quantitative RT-PCR experiments on MCF7 cells treated with ORG 2058. A. Southern blot performed with a HOXA1 cDNA probe after RT-PCR of HOXA1 mRNAs. B. Southern blot with $\beta$ actin used as an internal control for the semi-quantitative RT-PCRs. Size of HOXA1 and actin amplified fragments are illustrated. Hours of treatment are mentioned above the autoradiograms. 


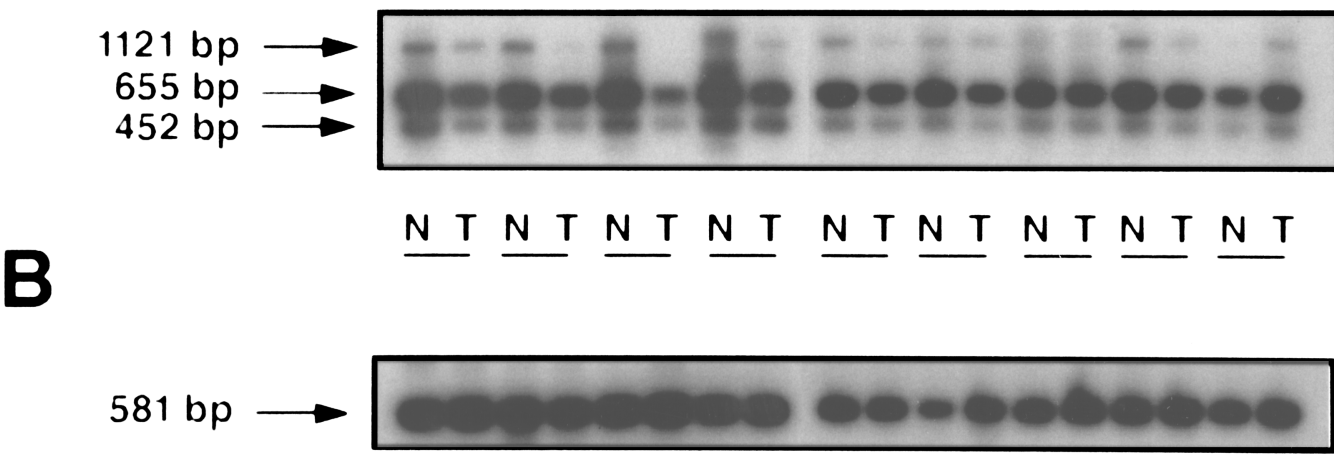

FIG. 4. A. Detection of HOXA1 expression in human breast cancer lesions (T) and adjacent tissues (N) by RT-PCR experiments. The resulting amplified fragments were visualized on a $1 \%$ ethidium bromide-stained agarose gel and submitted to Southern blot analysis. The three detected HOXA1 transcripts are indicated by arrows. B. Actin RT-PCR experiments performed on each sample. The resulting $581 \mathrm{bp}$ amplified fragment is illustrated by an arrow.

a role in malignant transformation and/or in tumor progression. Although it is too preliminary to extend these observations to the human HOXA1 gene, our results illustrating HOXA1 expression in MCF7 cells as well as in multiple breast cancer tissues may support this hypothesis. It is however not yet clear what role HOXA1 may play in breast cancer progression, and how modulation of the HOXA1 gene may affect that process. Based on HOXA1 induction by retinoic acid and progestins, we propose that HOXA1 may be one of the orchestrators that regulate breast epithelial cell differentiation.

\section{ACKNOWLEDGMENTS}

The authors are grateful to members of the laboratory for many helpful discussions and critical comments on the manuscript, to J. Gielen for his support and to E. Campo for providing us the breast cancer RNA samples. A.C. and V.C. are Research Assistant and Senior Research Associate of the National Fund for Scientific Research (Belgium), respectively.

\section{REFERENCES}

1. Liotta, L. (1986) Cancer Res. 46, 1-7.

2. Poste, G., and Fidler, I. J. (1980) Nature 283, 139-146.

3. Gehring, W. J., and Hiromi, Y. (1986) Ann. Rev. Genet. 20, 147-173.

4. Lewis, E. B. (1978) Nature 276, 565-570.

5. Levine, M., Rubin, G. M., and Tjian, R. (1984) Cell 38, 667-673.

6. Akam, M. (1989) Cell 57, 347-349.

7. Acampora, D., D’Esposito, M., Faiella, A., Pannese, M., Migliaccio, E., Morelli, F., Stornaiuolo, A., Nigro, V., Simeone, A., and Boncinelli, E. (1989) Nucleic Acid Res. 17, 10385-10402.

8. Lawrence, H. J., and Largman, C. (1992) Blood 80, 2445-2453.

9. Aberdam, D., Negreanu, V., Sachs, L., and Blatt, C. (1991) Mol. Cell. Biol. 11, 554-557.

10. Hatano, M., Roberts, C. W. M., Minden, M., Crist, W. M., and Korsmeyer, S. J. (1991) Science 253, 79-82.

11. Kamps, M. P., Murre, C., Sun, X-H., and Baltimore, D. (1990) Cell 60, 547-555.

12. Celetti, A., Barba, P., Cillo, C., Rotoli, B., Boncinelli, E., and Magli, M. C. (1993) Int. J. Cancer 53, $237-244$.

13. Cillo, C., Barba, P., Freschi, G., Bucciarelli, G., Magli, M. C., and Boncinelli, E. (1992) Int. J. Cancer 51, $892-897$.

14. De Vita, G., Barba, P., Odartchenko, N., Givel, J. C., Freschi, G., Bucciarelli, G., Magli, M. C., Boncinelli, E., and Cillo, C. (1993) Eur. J. Cancer 29A, 887-893.

15. Tiberio, C., Barba, P., Magli, M. C., Arvelo, F., Le Chevalier, T., Poupon, M. F., and Cillo, C. (1994) Int. J. Cancer 58, 608-615. 
16. Maulbecker, C. C., and Gruss, P. (1993) Cell Growth Differ. 4, 431-441.

17. Deguchi, Y., and Kehrl, J. H. (1993) Cancer Res. 53, 373-377.

18. Ee, H. C., Erler, T., Bhathal, P. S., Young, G. P., and James, R. J. (1995) Am. J. Pathology 147, 586-592.

19. Song, K., Wang, Y., and Sassoon, D. (1992) Nature 360, 477-481.

20. Suzuki, M., Tanaka, M., Iwase, T., Naito, Y., Sugimura, H., and Kino, I. (1993) Biochem. Bioph. Res. Co. 194, 187-193.

21. Chariot, A., Moreau, L., Senterre, G., Sobel, M. E., and Castronovo, V. (1995) Biochem. Biophys. Res. Com. 215, $713-720$.

22. Glisin, V., Crkvenjakov, R., and Byus, C. (1974) Biochemistry 13, 2633-2637.

23. Nakajima-Iijima, S., Hamada, H., Reddy, P., and Kakunaga, T. (1985) Proc. Natl. Acad. Sci. USA 82, $6133-6137$.

24. Siebert, P. D. (1994) Quantitative RT-PCR, Methods and Applications, Book 3, Clontech Laboratories Inc.

25. Itoh, F., Hinoda, Y., Ohe, M., Ohe, Y., Ban, T., Endo, T., Imai, K., and Yachi, A. (1993) Int. J. Cancer 53, $260-263$.

26. Simeone, A., Acampora, D., Nigro, V., Faiella, A., D’Esposito, M., Stornaiuolo, A., Mavilio, F., and Boncinelli, E. (1991) Mech. Dev. 33, 215-228.

27. Friedmann, Y., Daniel, C. A., Strickland, P., and Daniel, C. W. (1994) Cancer Res. 54, 5981-5985. 